\title{
LA AUTORREGULACIÓN EN EDUCACIÓN INFANTIL ES POSIBLE
}

Self-regulation in infant school is possible

A auto-regulação no jardim de infância é possível

Beatriz Castro Bayón (1)

Óscar Manuel Casado Berrocal (2)

(1) CEIP Vital Aza, +34 678402977. beatriz.castro.bayon@gmail.com.

(2) CEIP Ponce de León, Universidad Isabel I, +34 646920522.

oscar.casado.berrocal@gmail.com

\begin{abstract}
Resumen
La experiencia descrita aborda la autorregulación en la etapa de Educación Infantil,con la intención de fomentar la autonomía del alumnado desde las edades más tempranas. En esta estrategia metodológica los alumnos organizarán las actividades que realizarán cada día (todas ellas relacionadas con el proyecto trabajado) de manera lúdica y autónoma. Para ello, la docente proporciona a los alumnos unas tarjetas con pictogramas donde aparecen representadas las tareas a realizar cada día de la semana (lógicamatemática, psicomotricidad, juego libre, etc.). En la asamblea inicial de la jornada se explicarán todas las actividades a realizar y una vez finalizada, el alumno planificará su jornada de trabajo estableciendo el orden en el que desarrollará cada tarea, reflejando todo ello en el panelpara que puede ser evaluado.

Palabras clave: Educación Infantil; autorregulación; autonomía
\end{abstract}

\begin{abstract}
The experience described the self-regulation in infant school, with the intention of favoring the autonomy of students from the earliest ages. In thismethodologicalstrategy the students will organize the activities that will be carried out each day (all related to the project worked) in a playful and autonomous way. To do this, the teacher provides students with pictogram cards showing the tasks to be performed each day of the week (logic-mathematics, psychomotricity, free play, etc.). At the beginning of the day the teacher will explain all the activities to be carried out and once finished, the student will
\end{abstract}


plan his work deciding the order in which he will develop each task, reflecting all this in the panel so that it can be evaluated.

Keywords: Infant school; self-regulation; autonomy

\section{Resumo}

A experiência descrita endereços auto-regulação no jardim de infância, com a intenção de promover a autonomia dos alunos das primeiras idades. Na esta estratégia metodológica os alunos irão organizar as atividades realizadas todos os dias(todas relacionadas com o projeto trabalhou) em uma forma divertida e autónoma. Para fazer isso, o professor fornece aos alunos cartões com pictogramas, onde estão representadas as tarefas de cada dia da semana (lógico-matemática, psicomotor, jogo livre, etc.). Na reunião inicial do dia serão explicadas todas as atividades para realizar e após a conclusão, o aluno irá planejar seu dia de trabalho, definindo a ordem em que cada tarefa vai desenvolver, refletindo tudo no painel.

Palavras-chave: Jardim de infância; auto-regulação; autonomia

\section{Introducción}

Uno de los principales objetivos a conseguir en la etapa de Educación Infantil es que los alumnos logren "adquirir progresivamente autonomía en sus actividades habituales” (LOE, 2006 artículo 13.c). Para que el alumnado logre adquirir dicha autonomía, es fundamental que los docentes les demos las herramientas necesarias. En la etapa de Educación Infantil tenemos la oportunidad de comenzar a desarrollar la autonomía del alumnado, de cara a las etapas superiores, entendida ésta como la capacidad para desenvolverse libremente y con suficiencia, en situaciones cotidianas.

Por tanto, el tipo de tareas que debemos plantear en el aula deben generar situaciones en las que el alumnado tenga la opción de tomar decisiones sobre su aprendizaje, identificar los errores, aprender de ellos, compartir experiencias con otros compañeros, etc. Ejemplo de este tipo de propuestas son los rincones de trabajo (Laguia \& Vidal, 2011), el trabajo por proyectos (Díez Navarro, 1998) o los talleres en el aula (Trueba, 2000) entre otros. Todas estas metodologías quedarán completadas si además las llevamos a cabo a través de la autorregulación, gracias a la cual los alumnos podrán decidir y organizar cuándo realizar cada una de las tareas propuestas por el docente. 


\section{Contextualización}

La experiencia se está llevando a cabo actualmente con los alumnos de 4 años B (2a de Educación Infantil) del CEIP Vital Aza (Pola de Lena). Este grupo concreto está formado por 14 alumnos de 4 años de los cuales 8 son chicas y 6 chicos. Se trata de un grupo con diversidad cultural (provenientes de cuatro países diferentes) y con unas características psicoevolutivas muy dispares entre ellos.

\section{Diseño y desarrollo}

Debido a que el grupo con el que se lleva a cabo esta experiencia no había trabajado la autorregulación con anterioridad, se decide comenzar a aplicar a partir del $2^{\circ}$ trimestre, con el fin de conocer mejor las características de los alumnos.

En la etapa de Educación Infantil resulta primordial el fomento de la autonomía de los alumnos en clase, por lo que tras comprobar que el alumnado había adquirido las normas del aula y conocía dónde se encontraban los materiales y los rincones de trabajo de la misma, se decide comenzar con esta experiencia.

La estructura de trabajo es siempre similar y comienza explicando en la asamblea inicial del día las actividades que se van a realizar a lo largo de la jornada. El objetivo de esta iniciativa es lograr que los alumnos vayan implicándose en el proceso de aprendizaje, haciéndolo propio y por lo tanto, más adaptado a sus intereses y motivaciones. Esto favorecerá su capacidad para organizar y gestionar tareas a cortomedio plazo, algo sumamente importante conforme van avanzando los cursos y especialmente en las etapas superiores de Primaria y Secundaria (Casado, PérezPueyo\& Casado, 2017).Sin embargo, como toda novedad que se pretende poner en práctica con éxito, es necesario comenzar poco a poco, en situaciones muy facilitadas. Así pues, en un principio únicamente se pretende que los alumnos registren en un tablón los pictogramas de las tareas que han ido realizando (inmediatamente después de hacerlas y enseñárselas a la profesora).

Tras observar que ya en la primera semana todos los alumnos colocan autónomamente las tarjetas y conocen la dinámica, se decide dar un paso más: que sean los propios alumnos quienes se organicen y elijan en qué orden van a realizar las tareas a lo largo del día. Por tanto, se les pedirá que coloquen todos los pictogramas antes de 
realizar las tareas, es decir, justo después de que la maestra haya explicado las actividades de la mañana (Imagen 1).

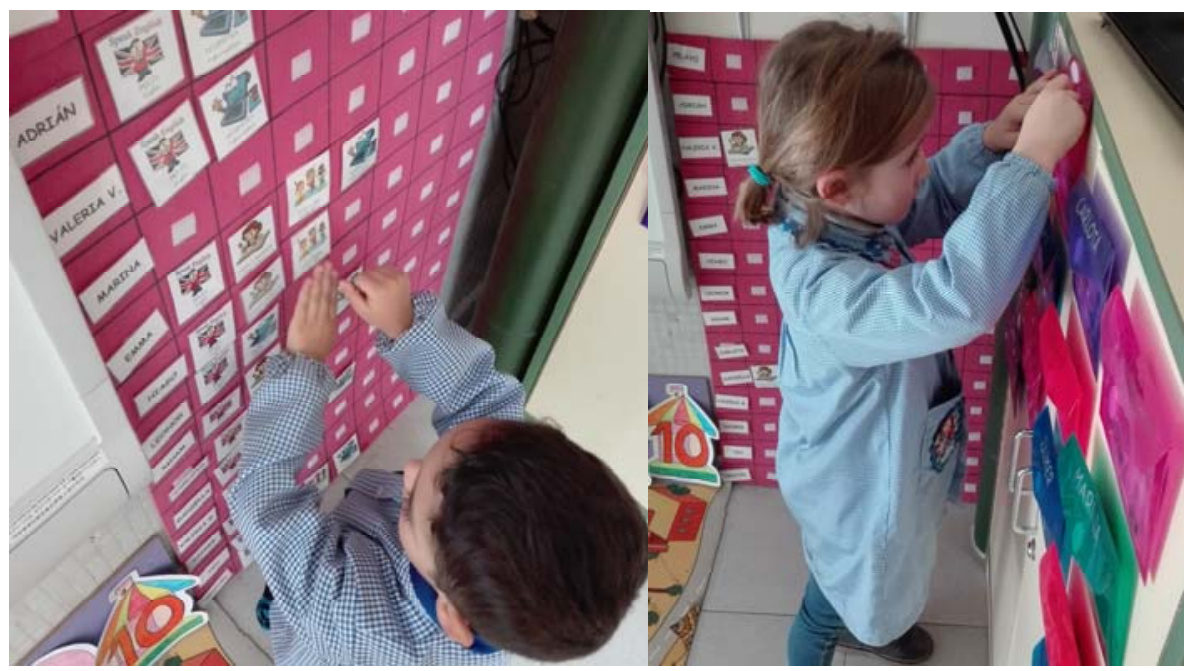

Imagen 1.

\section{Alumnos planificando las tareas de la mañana}

El panel se convertirá así en un horario simplificado que ellos mismos irán construyendo cada día al terminar la asamblea y que será diferente para cada uno (Imagen 2). Esto les permitirá anticiparse a su propia actuación e ir trabajando la noción de secuencia y linealidad temporal tan importante en la etapa de Educación Infantil.

La estructura de trabajo mencionada anteriormente se sigue manteniendo, solo que ahora, tras la pertinente explicación en la asamblea, cada alumno tendrá que organizar su mañana decidiendo el orden en el que realizará las tareas. Dichas tareas seguirán la estructura de trabajo por rincones de manera que no habrá problema en que varios alumnos coincidan haciendo la misma actividad.

Por otro lado, también es importante mencionar que la estructura que se ha aplicado permite seguir manteniendo la realización de rutinas con los alumnos (asamblea inicial y final,

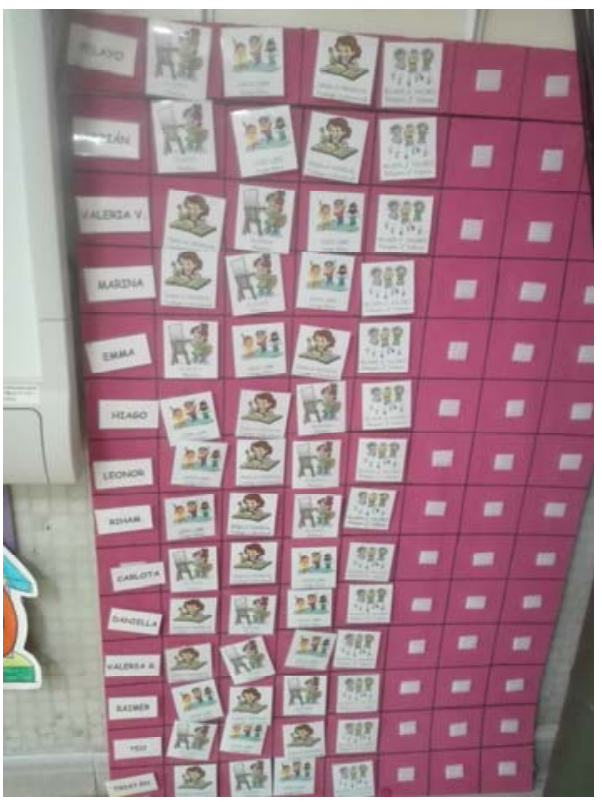

Imagen 2.

Panel con las actividades planificadas cuento, almuerzo, recreo, relajación, etc.), lo cual 
les otorga seguridad y les ayuda a conocer lo que viene a continuación. Tanto éstas como las áreas impartidas por especialistas (inglés, psicomotricidad, informática, religión/valores, etc.) se intercalarán con las tareas libres o de rincones que los alumnos habrán fijado en su plan diario.

Es importante explicar a los alumnos que estas actividades o periodos son fijos e inamovibles y que por lo tanto, no podrán ser ubicados libremente en su horario como sí pueden hacer con el resto de tareas.

\section{Evaluación y conclusiones}

Una vez puesta en práctica la experiencia, se ha observado que los alumnos la han acogido de manera muy positiva. Desde un primer momento han estado interesados acerca de cómo y cuándo hacer las tareas propuestas y ellos mismos iban razonando el porqué de su elección (ya que mientras algún alumno necesitaba jugar a primera hora de la mañana para estar luego más concentrado, otros preferían hacer antes todo el trabajo para poder después tener más tiempo para jugar). No obstante, también se observó que varios alumnos únicamente parecían estar interesados en la dinámica a la hora del juego, siendo necesario recordarles que también tenían que realizar otras tareas a lo largo de la jornada. Debido a que la experiencia descrita anteriormente ha resultado muy positiva tanto para el alumnado como para el profesorado implicado, se prevé su continuación al menos en el próximo curso para seguir fomentando la autonomía en el grupo clase.

\section{Referencias}

Casado, O.M., Pérez-Pueyo, A., \& Casado, P. (2017). La autorregulación en educación primaria. Una propuesta para favorecer la autonomía del alumnado. En LópezPastor, V. y Pérez-Pueyo, A. (coords.). Evaluación formativa y compartida en educación. León: Universidad de León.

Ley Orgánica 2/2006, de 3 de mayo, de Educación.

Trueba, B. (2000). Talleres integrales en Educación Infantil. Una propuesta de organización del escenario escolar. Madrid: Ediciones de la Torre.

Díez Navarro, C. (1998).La oreja verde de la escuela. Madrid: Ediciones de la Torre.

Laguia, M.J., \& Vidal, C. (2011). Rincones de actividad en la escuela infantil (0 a 6 años). Barcelona: Graó. 